\title{
Unusual thermal boundary resistance in halide perovskites: A way to tune ultralow thermal conductivity for thermoelectrics
}

Tianjun Liu,,$^{\dagger, \#}$ Sheng-Ying Yue, ${ }^{t, j}, \#$ Sinclair Ratnasingham, ${ }^{\S}$ Thibault Degousée, ${ }^{\dagger}$

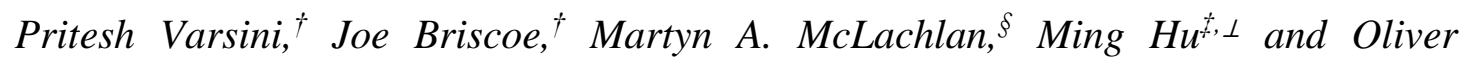
Fenwick $^{\dagger, *}$

${ }^{\dagger}$ School of Engineering and Materials Science, Organic Thermoelectrics Laboratory, Materials Research Institute, Queen Mary University of London, Mile End Road, London E1 4NS, UK.

\$Institute of Mineral Engineering, Division of Materials Science and Engineering, Faculty of Georesources and Materials Engineering, Aachen Institute for Advanced Study in Computational Engineering Science (AICES), RWTH Aachen University, 52062 Aachen, Germany.

"Department of Mechanical Engineering, University of California, Santa Barbara, CA, 93106, USA.

${ }^{\S}$ Department of Materials and Centre for Plastic Electronics, Imperial College London, London SW7 2AZ, UK.

${ }^{\perp}$ Department of Mechanical Engineering, University of South Carolina, Columbia, SC 29208, USA

KEYWORDS: Halide perovskite, phonon, in-plane, thermal conductivity, thermal transport, thermoelectrics. 


\section{Abstract}

Halide perovskites have emerged as promising candidates as the active material in photovoltaics and light-emitting diodes. They possess unusual bulk thermal transport properties that have been the focus of a number of studies, but there is much less understanding of thermal transport in thin films where a diverse range of structures and morphologies are accessible. Here, we report on the tuning of in-plane thermal conductivity in methylammonium lead iodide thin films by morphological control. Using 3- $\omega$ measurements, we find that the room temperature thermal conductivity of thermally evaporated methylammonium lead iodide perovskite films ranges from 0.31 to $0.59 \mathrm{~W} / \mathrm{mK}$. We measure a discontinuity in thermal conductivity at the orthorhombic-tetragonal phase transition and explore this using density functional theory, and attributing it to a collapse in the phonon group velocity along the $c$-axis of the tetragonal crystal. Moreover, we have quantified the thermal boundary resistance (Kapitza resistance) for thermally evaporated films, allowing us to estimate the Kapitza length which is $36 \pm 2 \mathrm{~nm}$ at room temperature and $15 \pm 2 \mathrm{~nm}$ at $100 \mathrm{~K}$. Curiously, the Kapitza resistance has a strong temperature dependence which we also explore using density functional theory, with these results suggesting an important role of methylammonium rotational modes in scattering phonons at the crystallite boundaries. 
Halide perovskites have been recognized as promising photovoltaic (PV) materials, ${ }^{1-3}$ due to their large absorption coefficients, high charge carrier mobilities, ${ }^{4}$ and large charge carrier diffusion lengths. ${ }^{5}$ On top of this they can be grown as single crystals, ${ }^{6}$ or deposited as polycrystalline films by a variety of techniques including vapour phase deposition, ${ }^{7}$ and solution methods compatible with large area printing. Despite intense research on halide perovskite materials for PVs, and a growing body of research on their use in field-effect transistors ${ }^{8,9}$ and light-emitting diodes, ${ }^{10-12}$ there have only been a small number of studies on their thermoelectric properties. ${ }^{13,14}$

Efficient thermoelectric materials possess a high figure of merit, $Z T=\sigma S^{2} T / \kappa$, where $\mathrm{T}$ is the temperature and $\sigma, \mathrm{S}$ and $\kappa$ are the electrical conductivity, Seebeck coefficient and thermal conductivity respectively. A number of studies have indicated that methylammonium lead iodide $\left(\mathrm{MAPbI}_{3}\right)$ possesses a large Seebeck coefficient ${ }^{14,15}$ and high charge carrier mobility, ${ }^{16}$ with others highlighting a remarkably low thermal conductivity in single crystals, ${ }^{15,}{ }^{17}$. These materials, which compositionally can be considered as organic-inorganic hybrids, can therefore, in certain terms, be considered organic-inorganic hybrids in terms of their thermoelectric properties, i.e. sharing high charge mobilities associated with inorganic materials and intrinsically low thermal conductivity and solution processability usually associated with organic materials. This has fueled speculation that the halide perovskites might be excellent thermoelectric materials. Correspondingly, single-crystal caesium tin iodide perovskite nanowires have been reported with a promising figure of merit $(Z T=0.11$ at $320 \mathrm{~K})$ which includes a low lattice thermal conductivity $(0.38 \pm 0.04 \mathrm{~W} / \mathrm{mK}) .{ }^{13}$ However, to achieve a figure 
of merit suitable for applications ( $\mathrm{ZT}>1$ ), much more needs to be understood about how to simultaneouslteously tune $\sigma, \mathrm{S}$ and $\kappa$ in these materials.

Understanding thermal transport in halide perovskites is therefore of critical importance for thermoelectrics, ${ }^{18}$ but also has consequences for heat dissipation in other devices such as field effect transistors (FETs), light-emitting diodes (LEDs) and PVs. Hata et al. ${ }^{19}$ found, by means of nonequilibrium molecular dynamics, that rotations of methylammonium cations are responsible for phonon transport suppression. Wang et $a l .{ }^{20}$ attributed the low thermal conductivity to low phonon group velocities caused by low elastic stiffness, in addition to short phonon lifetimes $(<100 \mathrm{ps})$ and mean free paths $(<10 \mathrm{~nm})$ due to enhanced phonon-phonon scattering.

Despite the low bulk lattice thermal conductivity already reported for these materials, it remains to be seen whether there is much scope to reduce the thermal conductivity below bulk values, and understanding of how morphology and grain/domain boundaries can be used to tune the thermal conductivity still needs to be developed. In this study, we analyse the in-plane thermal conductivity of $\mathrm{MAPbI}_{3}$ thin films as a function of temperature $(100 \mathrm{~K}-300 \mathrm{~K})$, spanning the orthorhombic and tetragonal phases and measuring across the phase transition between the two at $160 \mathrm{~K}$. We selected $\mathrm{MAPbI}_{3}$ as the system to study in part because its bulk thermal conductivity has already been the focus of computational study by several groups, ${ }^{17,20}$ including by us, ${ }^{21}$ enabling us to test the assumptions of those models. The tin halide perovskites yield higher thermoelectric performance and higher conductivity $\left(>200 \mathrm{Scm}^{-122}\right)$ which would enable study of competing effects on electrical and thermal conductivity at 
interfaces, which is important in the study of thermoelectric properties, but these materials are not stable in air for more than a few minutes. On the other hand, extrinsic doping of the more stable lead halide materials has been studied recently but so far yields conductivities orders of magnitude lower than the tin halide perovskites. ${ }^{23,24}$ For this reason we focus this study on the lattice thermal properties in (non-conductive) $\mathrm{MAPbI}_{3}$ thin films to enable us to unambiguously elucidate the impact of nanostructure on thermal conductivity within the stability window of the materials. Since grains in $\mathrm{MAPbI}_{3}$ typically span the full film thickness, but with finite lateral size, our in-plane measurements are particularly sensitive to grain boundaries. By tuning the crystallite size of thermally evaporated thin films, thermal conductivity ranging from $\sim 0.3$ to $\sim 0.6$ $\mathrm{W} / \mathrm{mK}$ was measured. We quantify the thermal boundary resistance (Kapitza resistance) in polycrystalline films for the first time and discover that it is temperature dependent. We explore the nanoscopic origins of this effect by means of density functional theory (DFT). We investigate further morphologies in the form of aerosolassisted chemical vapour deposited (AACVD) films finding even lower thermal conductivities $(\sim 0.12 \mathrm{~W} / \mathrm{mK}$ at $300 \mathrm{~K})$ and observing a discontinuity in thermal conductivity at the orthorhombic-tetragonal phase transition, which we explain by a collapse in the phonon group velocity along the $b$-axis of the crystal.

\section{RESULTS AND DISCUSSION}

$\mathrm{MAPbI}_{3}$ thin films were deposited by co-evaporation of lead chloride and methylammonium iodide. As shown in Figure 1, X-ray diffraction (XRD) data of 
$\mathrm{MAPbI}_{3}$ films of three different thicknesses $(65 \pm 3 \mathrm{~nm}, 80 \pm 5 \mathrm{~nm}$ and $100 \pm 12 \mathrm{~nm})$, all show peaks at $14.0^{\circ}$ and $28.1^{\circ}(2 \theta)$ corresponding to (110) and (220) planes of the tetragonal $\mathrm{MAPbI}_{3}$ crystal structure, respectively ${ }^{3,25,26}$. The $\mathrm{MAPbI}_{3}$ (330) peak at $43.2^{\circ}$ is also visible in the thicker films, whilst none of the samples show the characteristic peak of $\mathrm{MAPbCl}_{3}$ at $15.7^{\circ} \cdot{ }^{27}$ On the other hand, the (001) peak of $\mathrm{PbI}_{2}$ at $12.4^{\circ}$ is clearly visible in the thinnest films, which is a sign of an incomplete surface reaction, for which $\mathrm{PbI}_{2}$ is an intermediate product often observed in thin films deposited by this method. ${ }^{7}$ The $\mathrm{PbI}_{2}$ content decreases as the film thickness (and hence reaction time) increases. Scanning electron microscopy reveals the typical polycrystalline morphology of our vapour-deposited $\mathrm{MAPbI}_{3}$ thin films (Figure 1c-e), indicating a grain size which increases with film thickness.

The thermal conductivity of these $\mathrm{MAPbI}_{3}$ thin films (Figure 2b) at room temperature is $0.31 \pm 0.03 \mathrm{~W} / \mathrm{mK}, 0.44 \pm 0.03 \mathrm{~W} / \mathrm{mK}$ or $0.59 \pm 0.04 \mathrm{~W} / \mathrm{mK}$ for film thicknesses of $65 \pm 3 \mathrm{~nm}, 80 \pm 5 \mathrm{~nm}$ and $100 \pm 12 \mathrm{~nm}$ respectively. These values are broadly in line with those reported by Pisoni et al. ${ }^{28}(0.5 \mathrm{~W} / \mathrm{mK}$ in single crystals and $0.3 \mathrm{~W} / \mathrm{mK}$ in polycrystalline pellets $)$ and Mettan et al. ${ }^{14}(0.5 \pm 0.1 \mathrm{~W} / \mathrm{mK}$ in single crystals). We note that the thermal conductivity of $\mathrm{PbI}_{2}(\sim 2 \mathrm{~W} / \mathrm{mK}$ for single crystals and in the out-of-plane direction in thin films ${ }^{29,30}$ ) is much higher than our measured values, so we can infer that increased phonon scattering at grains and domain boundaries in the thinner films is the dominant effect over the higher thermal conductivity contribution of $\mathrm{PbI}_{2}$ inclusions. To verify this, we measured the the thermal conductivity of a $135 \pm 5 \mathrm{~nm}$ thermally evaporated polycrystalline $\mathrm{PbI}_{2}$ thin 
film, finding a room temperature thermal conductivity of $0.51 \pm 0.02 \mathrm{~W} / \mathrm{mK}$, that is significantly below the intrinsic value.

To explore alternative morphologies and larger crystallite sizes, we deposited $\mathrm{MAPbI}_{3}$ films by aerosol-assisted chemical vapour deposition (AACVD), detailed in the experimental section at the end of the manuscript. In this technique, which we reported previously, ${ }^{31}$ the $\mathrm{MAPbI}_{3}$ precursors $\left(\mathrm{PbI}_{2}\right.$ and $\left.\mathrm{MAI}\right)$ are delivered to the heated substrate sequentially in aerosols of the carrier solvents dimethyl formamide (for $\mathrm{PbI}_{2}$ ) and methanol (for MAI). Initially a $\mathrm{PbI}_{2}$ film is formed on the surface and then the MAI is delivered, reacting with $\mathrm{PbI}_{2}$ to form the final film. This is a scalable process allowing deposition of thick films (many microns), with large grains (microns in diameter) typically spanning the full thickness of the film. XRD indicates that there is no residual $\mathrm{PbI}_{2}$ in films produced by this method. The increased mass of material in our films improved the sensitivity of our measurements and enabled us to clearly observe the orthorhombic to tetragonal phase transition as a discontinuity in the thermal conductivity at $\sim 160 \mathrm{~K}$ (Figure $2 \mathbf{2 d}$ ). This manifests itself as a 5-8 \% decrease in the thermal conductivity going from the low temperature (orthorhombic) phase to the higher temperature (tetragonal) phase. Accompanying the decrease in the absolute value of the thermal conductivity is a discontinuity in the gradient, $d \kappa / d T$. These observations are in line with the literature. ${ }^{14,29}$ The low absolute value of thermal conductivity of our AACVD films can be understood in terms of morphology, since the roughness of these films is comparable to the film thickness. This implies a poor connectivity between grains in the in-plane direction (the measurement direction). 
Grains are only in thermal contact to one another within a thin region close to the substrate creating a bottleneck to thermal transport. The thickness of the region of good thermal contact between grains in this case is much less than the average thickness used in the calculation of thermal conductivity. For thicker AACVD films there is more time during film formation for grains to coalesce, and in these cases we observe that the thermal conductivity increases towards the values of thermally evaporated films (Supporting Information).

To understand the discontinuity in thermal conductivity at the phase transition we performed first-principles calculations to examine the phonon modes in each phase. ${ }^{32-}$ ${ }^{36}$ In previous work ${ }^{37}$ we demonstrated that only the low-frequency phonons belonging to the inorganic cage contribute to thermal transport process in $\mathrm{MAPbI}_{3}$. The group velocities of modes on the MA cation are effectively zero, but MA modes can still interact with the propogating modes by acting as scattering centres. However, our previous simulations ${ }^{21}$ showed that only motions of the entire MA cation couple to the cage modes, and not internal motions (i.e. vibrations and torsions) of the MA molecule. In that work, we calculated the phonon modes relevant to thermal conductivity in $\mathrm{MAPbI}_{3}$ by considering vibrations of the $\mathrm{Pb}$-I cages and rotational modes of the MA molecule as whole, but freezing the inner freedoms belonging to the methylammonium (MA) molecule. Here we adopt the same method to calculate phonon modes of the orthorhombic and tetragonal phases. Figure 3a-d presents the phonon dispersion curves of two phases, their phonon density of states (DOS) and the cumulative integral of the phonon DOS. 
We also calculated the group velocities, $v_{g}$, of the low frequency phonons belonging to the $\mathrm{Pb}$-I cages. The group velocities projected on the $a, b$ and $c$-directions of the primitive cells are shown in Figure 4. The $b$-direction of the orthorhombic phase is equivalent to the $c$-direction of the tetragonal phase. Our data show a collapse in the group velocities along this crystal direction when moving from the orthorhombic to tetragonal phase, meaning that the phonon transporting ability reduces when the structural phase transition occurs. In general, the total phonon thermal conductivity can be described as $k=\frac{1}{3} C{\overline{v_{g}}}^{2} \tau$, where $C$ is heat capacity, $\overline{v_{g}}$ is group velocity and $\tau$ is phonon lifetime. Indeed our DFT calculations imply a sharp reduction in in $v_{g}$ across the orthogonal to tetragonal phase transition (Figure 4a,b). Our modelling presented in the Supporting Information shows that heat capacity is continuous across the phase transition, so does not contribute to this discontinuity in thermal conductivity. On the other hand, the phonon lifetimes in $\mathrm{MAPbI}_{3}$ have previously been measured from Raman line widths, ${ }^{38}$ with the finding that $\tau$ decreases sharply at the phase transition from orthorhombic to tetragonal for some of the phonon modes. Putting this together, there are two mecahnisms identified for the sharp decrease in thermal conductivity going from the orthogonal to tetragonal phase, which we do indeed observe in our own thermal conductivity measurements.

Thermal transport as a function of crystalline dimensions is important in polycrystalline materials because of strong phonon scattering at grain and domain boundaries which introduces a thermal resistance between crystallites ${ }^{39-41}$ known as a Kapitza resistance ${ }^{42-44}, R_{k}$. This effect can be particularly important in thin 
polycrystalline films where the overall film thickness provides an upper limit to the crystal sizes. There is, therefore, a functional dependence of the thermal conductivity on crystallite size, $d$, which is mediated by the Kapitza resistance: ${ }^{43}$

$$
\kappa(T, d)=\frac{\kappa_{i}(T)}{1+\frac{R_{k}(T) \kappa_{i}(T)}{d}} \text { equation } 1
$$

The intrinsic thermal conductivity of the material in the absence of crystallite boundaries is given by $\kappa_{i} . R_{k}$ and $\kappa_{i}$ can therefore be determined by a linear fit of the experimental data for $\kappa^{-1}$ against $d^{-1}$ at each temperature, where the crystallite size is estimated by the Scherrer equation (details in Supporting Information). This model has been applied in yttria-stabilized zirconia ${ }^{41}$ (YSZ), bulk $\mathrm{Mg}_{3} \mathrm{Sb}_{2}{ }^{45}$ and polycrystalline $\mathrm{Si}_{\mathrm{x}} \mathrm{Ge}_{1-\mathrm{x}}{ }^{43}$ amongst others, and since $\kappa$ is a macroscopic measurement $R_{k}$ represents an ensemble average of the boundaries within the matieral. We employed this model to calculate the intrinsic thermal conductivity and Kapitza resistance of the thermally evaporated $\mathrm{MAPbI}_{3}$ thin films with our results shown in Figure 5 (details of calculations in the Supporting Information). From $100 \mathrm{~K}$ to $150 \mathrm{~K}$, the values of $R_{k}$ are approximately constant at $\sim 0.8 \times 10^{-8} \mathrm{~m}^{2} \mathrm{~K} / \mathrm{W}$. There is then a steady increase in $R_{k}$ with increasing temperature up to a value of $\sim 4 \times 10^{-8} \mathrm{~m}^{2} \mathrm{~K} / \mathrm{W}$ at $\sim 270 \mathrm{~K}$ (Figure 5a). In many other semiconductor systems, ${ }^{43,}, 4-48 \quad R_{k}$ has been found to be almost temperature independent, but in at least one case, an increase with temperature was observed $^{45}$ and this was attributed to some damage and/or disorder at grain boundaries at high temperature. A macroscopic description of the positive temperature correlation of $R_{k}$ for $\mathrm{MAPbI}_{3}$ thin films may have similar origins either in damage or reversible 
changes to the thermal contact between crystallites caused by anomalous thermal expansion effects, i.e. a thermal expansion expressed in the direction perpendicular to the plane of the thin film that causes a decrease in the contact area between crystallites in the in-plane direction. However, an alternative atomic-level description comes out of our computational model.

The upper limits of the phonon frequency belonging to the Pb-I cages is $\sim 125 \mathrm{~cm}^{-1}$ (associated temperature $\sim 180 \mathrm{~K}$ ) for the both phases (Figure 3). When the temperature is larger than $\sim 180 \mathrm{~K}$ (in the tetragonal phase), all the phonons belonging to the $\mathrm{Pb}-\mathrm{I}$ cages will be excited. This is reflected in the phonon heat capacity of $\mathrm{MAPbI}_{3}$ (Supporting Information), which tends to a constant value at this temperature. As the temperature is increased above $\sim 180 \mathrm{~K}$, the phonon modes of the collective motion belonging to the $\mathrm{MA}^{+}$molecule will be excited, which includes the rotational modes and translational modes with frequencies of $\sim 4 \mathrm{THz} .{ }^{37}$ Although these MA modes do not contribute to the thermal transport directly, their anharmonicity will provide scattering processes to the propagating phonon modes of $\mathrm{Pb}$-I cages. We note that phonons associated with internal motions of the MA cations reside in the range 25 $50 \mathrm{THz}$, and this huge energy gap disables the phonon scattering between the internal motions of the MA and the lattice vibrations of the $\mathrm{Pb}$-I cages. In our previous research, ${ }^{37}$ we found that the rotational modes of MA have larger anharmonicity than the translational modes and are therefore more likely to scatter phonons. This anharmonicity combined with the correlation of the onset of rotational MA modes with 
the onset of the increase in $R_{k}$, strongly indicates a role of MA rotations in the high boundary resistance at room temperature.

Our estimation of intrinsic thermal conductivity at $300 \mathrm{~K}, \kappa_{i}=0.68 \pm 0.15 \mathrm{~W} / \mathrm{mK}$ is at the high end of previously reported experimental values for bulk crystals $\left(\kappa_{i, b u l k}\right.$ $=0.5 \mathrm{~W} / \mathrm{mK})^{15,28,49}$. This discrepancy can be attributed to larger defect densities in bulk crystals, whereas defects can easily be ejected to boundaries in the smaller crystals found in thin film samples such as our own. To further quantify the crystallite-size dependence of $\kappa$ for a given material, the Kapitza length, $L_{k}=R_{k} \kappa_{i}$, was calculated as $36 \pm 2 \mathrm{~nm}$ at room temperature and $15 \pm 2 \mathrm{~nm}$ at $100 \mathrm{~K}$. Only for $\mathrm{d}>>L_{k}$, can $\kappa$ approach $\kappa_{i}$. Interestingly, our data shows (Figure 5c) that nanostructuring $\mathrm{MAPbI}_{3}$ in the range $5 \mathrm{~nm}-100 \mathrm{~nm}$ can drastically reduce the thermal conductivity, predicting a remarkably low thermal conductivity of $0.30 \pm 0.04 \mathrm{~W} / \mathrm{mK}$ at room temperature when $\mathrm{d}=10 \mathrm{~nm}$, or $0.16 \mathrm{~W} / \mathrm{mK}$ at room temperature when $\mathrm{d}=5 \mathrm{~nm}$. Crystallite size tuning to these length scales is achievable in halide perovskites through control of deposition conditions ${ }^{50}$ or by exploiting self-assembly processes using molecular additives. ${ }^{51}$ This result reveals the important role of nanostructure in thermal management strategies for halide perovskites devices.

\section{CONCLUSION}

Our work has shown that the thermal conductivity of lead halide perovskites thin films can be reduced below the already low bulk values by fine tuning of the morphology. The morphologies we studied using thermal evaporation and aerosol-assisted chemical 
vapour deposition span two orders of magnitude in crystallite size and film thickness. We have observed a discontinuity in the thermal conductivity at the orthorhombic to tetragonal phase transition which can be explained by a collapse in the phonon group velocity along one of the crystal axes. A peculiar increase in the Kapitza resistance that coincides with the thermal excitation of MA rotational modes, suggests an important role of these modes in determining the room temperature Kapitza resistance. Our measurements of Kapitza resistance point to a strategy to achieve ultralow thermal conductivity $(<0.2 \mathrm{~W} / \mathrm{mK})$ by tuning crystallite size to $\sim 5 \mathrm{~nm}$, which could be useful in the optimisation of halide perovskites as thermoelectric materials.

This work has therefore presented a potentially useful route to improving thermoelectric performance in halide perovskite materials, which is already predicted to achieve ZT 1-2 in bulk crystals. ${ }^{52}$ However, interfaces will only boost the thermoelectric figure of merit, $Z T$, if they decrease thermal conductivity without significantly degrading the electrical conductivity. The choice of a non-conductive halide perovskite in this work enabled a detailed study of the effects of morphology on lattice thermal conductivity on a reasonably stable halide perovskite material. Electrical conductivity can exceed $200 \mathrm{Scm}^{-1}$ in tin halide perovskites, ${ }^{22}$ but these materials are unstable in air. Strategies to electrically dope more stable halide perovskite systems are underdeveloped, and questions about whether nanostructuring can boost ZT will probably remain speculative until stable conductive systems have been synthesized.

\section{METHODS}


Vapur deposition of thin films. $\mathrm{MAPbI}_{3}$ films were produced by the coevaporation of lead chloride and methyl ammonium iodide at $10^{-7} \mathrm{mbar}^{\mathrm{PbCl}} \mathrm{Pb}_{2}$ and MAI are known to react on the sample surface to form $\mathrm{MAPbI}_{3}$ under conditions of excess MAI. $^{7}$ The deposition rate was $5.0 \AA$ A/s for methylammonium iodide (MAI, achieved with a crucible temperature of $\sim 120^{\circ} \mathrm{C}$ ) and $1 \AA / \mathrm{s}$ for $\mathrm{PbCl}_{2}$ (achieved with a crucible temperature of $\sim 320^{\circ} \mathrm{C}$ ). All chemicals purchased from commercial company without purification. The vacuum thermal evaporator opens directly into the glovebox enabling transfer without contact with air to where they were stored until measurement. Transfer of the samples to the Linseis Thin Film Analyzer was done with less than 2 minutes contact with air before pumping down of the test chamber to vacuum $\left(\sim 10^{-7} \mathrm{mbar}\right)$.

Aerosol-assisted chemical vapour deposition of micron-thick films. $\mathrm{PbI}_{2}(99 \%)$ was dissolved in the carrier solvent dimethyl formamide (>>99\%) at $0.1 \mathrm{~g} / \mathrm{mL}$. Methyl ammonium iodide (MAI, Solaronix) was dissolved in methanol (anhydrous, 99.8\%) at $0.033 \mathrm{~g} / \mathrm{mL}$. The precursor mists were created using an ultrasonic humidifier. The DMF:PbI ${ }_{2}$ mist was delivered to the reaction chamber with nitrogen gas at a flow rate of $0.75 \mathrm{~L} \mathrm{~min}^{-1}$ where the substrate was heated to $75^{\circ} \mathrm{C}$. The methanol:MAI mist was delivered to the reaction chamber with a nitrogen gas flow rate of $0.3 \mathrm{~L} \mathrm{~min}^{-1}$ with the substrate heated to $220{ }^{\circ} \mathrm{C}$. The perovskite films were removed from the reaction chamber after deposition and transferred immediately to a nitrogen glove box, where they were stored before further analysis. Further details of this technique have been reported previously. ${ }^{31}$ 
Morphology characterization and crystallography. SEM images were recorded by an FEI Inspect-F scanning electron microscopy. X-ray diffraction was performed by Siemens D5000 X-Ray Powder diffraction using a Cu Ka source $(\lambda=1.54 \AA)$.

Thermal conductivity measurement. The $\mathrm{MAPbI}_{3}$ thin films were deposited directly onto a pre-patterned test chip. The test chip (Linseis Messgeräte GmbH) comprises of a silicon substrate incorporating a suspended $\mathrm{Si}_{3} \mathrm{~N}_{4}$ membrane with integrated thin-film heater for in-plane measurement of the thermal conductivity by the 3- $\omega$ method in the Völklein geometry ${ }^{53-55}$. The surface termination of the test chip is a $30 \mathrm{~nm}$ thick layer of aluminium oxide. The measurement geometry is detailed in Supporting information. All the samples were prepared in a thermal evaporator housed in a nitrogen glovebox. The vacuum in the thermal evaporator was $\sim 10^{-7}$ mbar prior to deposition, and the oxygen and water levels in the glovebox $<0.1 \mathrm{ppm}$. After deposition, the samples were rapidly transferred from the glovebox to the characterisation instrument, during which time they were exposed to air for a maximum of 1 minute. The thermoelectric characterisation equipment was pumped down to 10-7 mbar immediately after loading samples.

Density functional theory (DFT) method. The phonon modes of $\mathrm{MAPbI}_{3}$ were calculated via the finite displacement difference method implemented in the PHONOPY package ${ }^{32}$. We calculated the phonon dispersion based on density functional theory using VASP code package. The Perdew-Burke-Ernzerhof parametrization of the generalized gradient approximation (GGA) ${ }^{33}$ was applied for the exchange-correlated functional, and the projector-augmented wave (PAW $)^{34,35}$ method 
was used to model the core electrons (for $\mathrm{Pb}$ the $5 \mathrm{~d}$ orbitals are included). The energycutoff of plane wave was set as $700 \mathrm{eV}$. For the parameter of the partial occupancies we adopted the Gaussian smearing and the width of the smearing was $0.01 \mathrm{eV}$. The van der Waals (vdW) interactions were also considered here as non-bonded terms ${ }^{36}$. When we calculated the low-frequency phonon dispersion of the orthorhombic and tetragonal phases, we considered the whole MA molecule as a rigid object, meaning that we froze its inner freedoms. The unit cell of the orthorhombic and tetragonal phases contains 48 atoms. We adopted $2 \times 2 \times 2$ supercell for the both phases and used $6 \times 6 \times 6$ Monkhorst-Pack grids as k-point sampling. The phonon group velocities $\left(\mathrm{v}_{g}\right)$ can be obtained by $v_{g}=\frac{d \omega}{d k}$, where $\omega$ is the phonon frequency and $k$ is the momentum vector of phonons in $k$ space. Simultaneously, we obtain the partial density of states (PDOS) and the free energy, the inner energy, the entropy and the phonon heat capacity with PHONOPY package ${ }^{32}$. 
$\mathbf{a}$

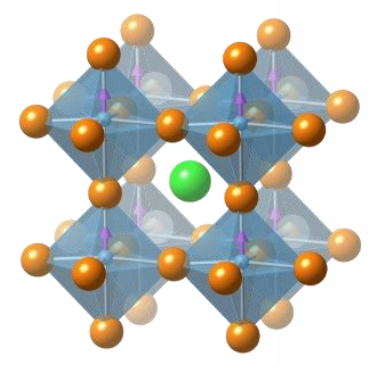

c

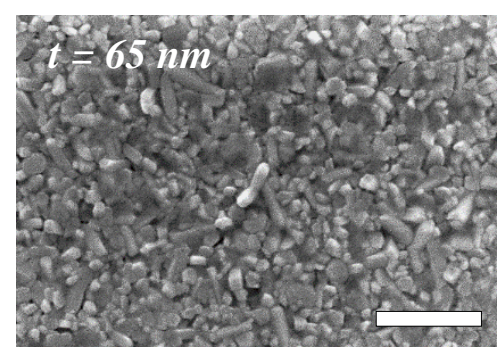

b
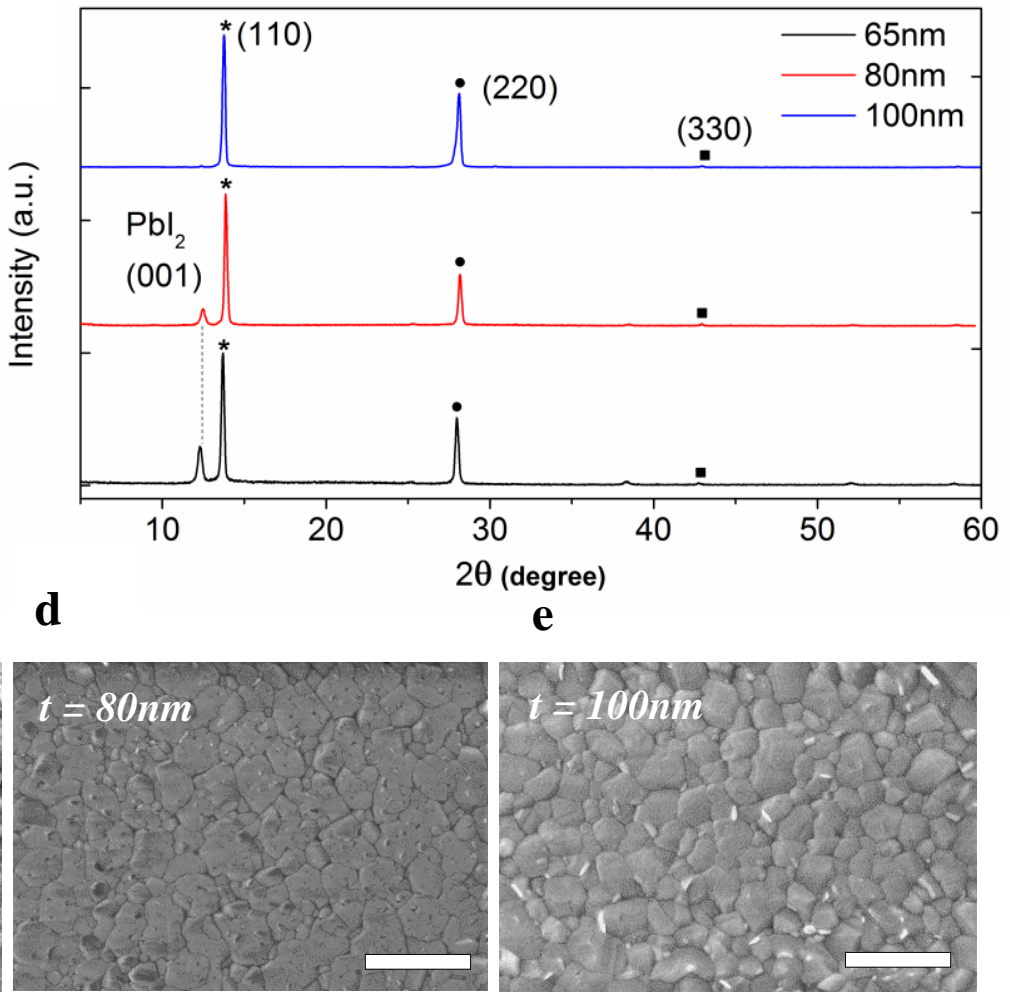

$\mathbf{e}$

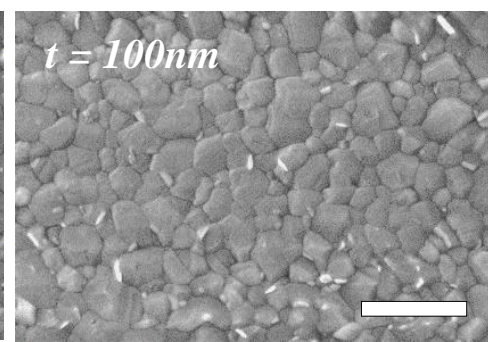

Figure 1. Perovskite thin film morphology and structure. (a) Crystal structure of $\mathrm{MAPbI}_{3}$. Species are coloured MA: green, Pb: blue, I: brown. (b) XRD patterns of perovskites thin films of $65 \mathrm{~nm}$ (black), 80nm (red) and 100nm (blue) thickness. Stars, circles and squares represent the (110), (220) and (330) planes of the perovskite crystal structure, respectively. (c) - (e) Scanning electron microscopy (SEM) images of $65 \mathrm{~nm}, 80 \mathrm{~nm}$ and $100 \mathrm{~nm}$ thick perovskite thin films. The scale bars are $500 \mathrm{~nm}$. 


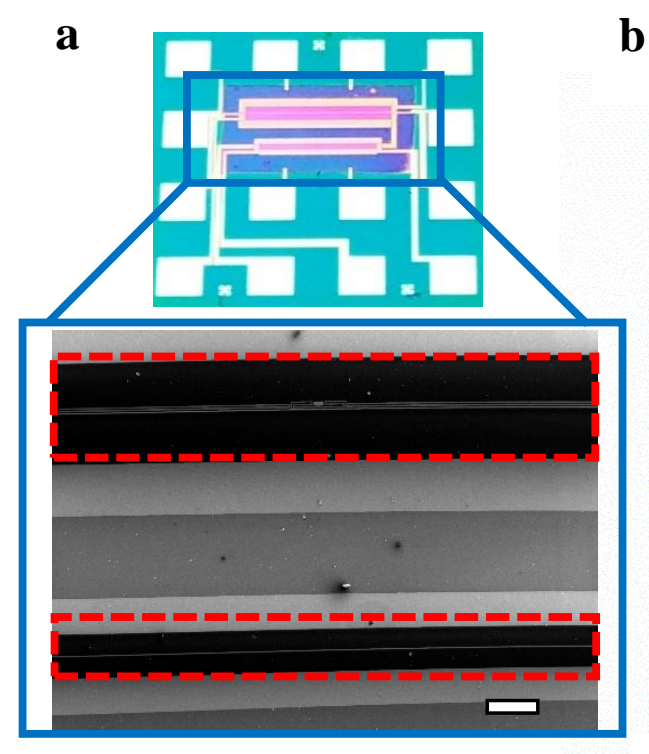

c

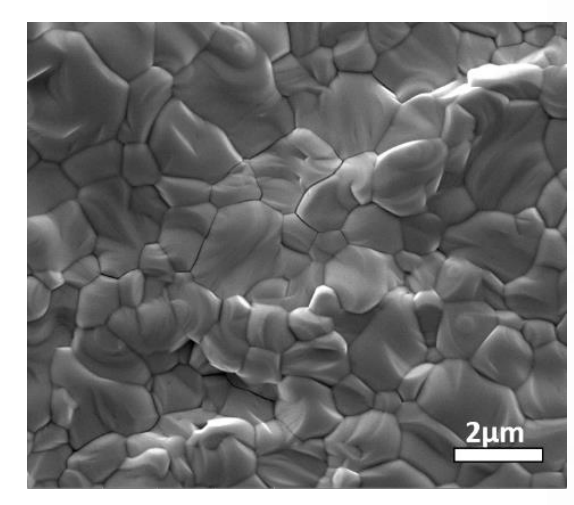

b

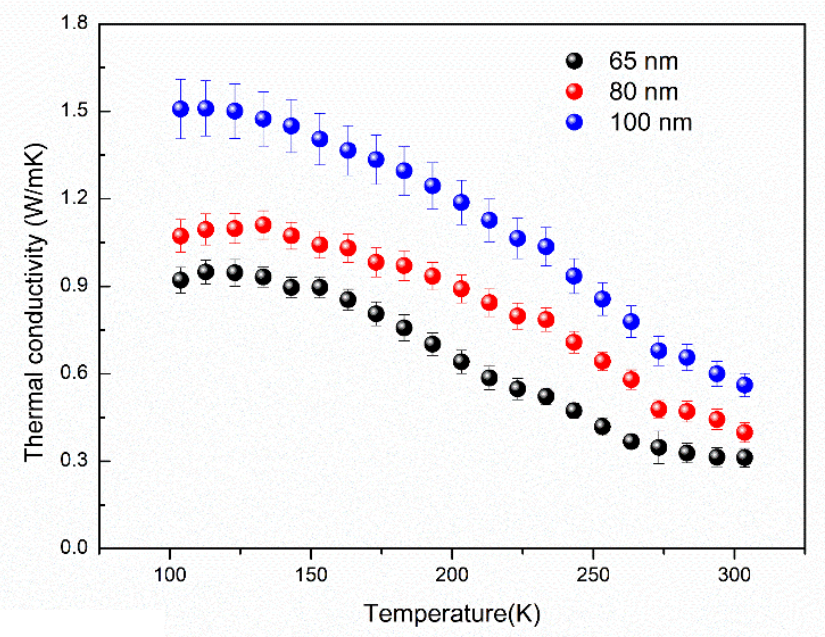

d

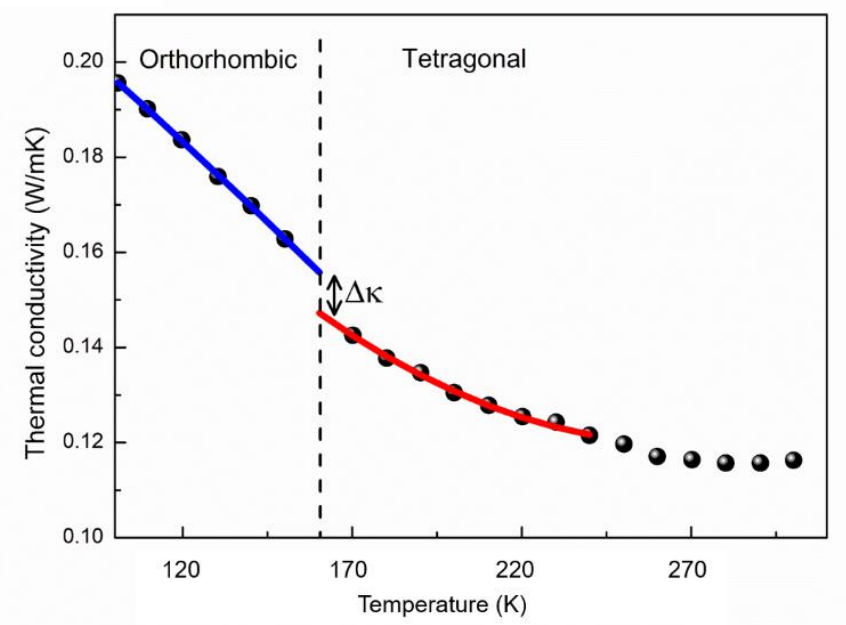

Figure 2. Thermal conductivity of $\mathrm{MAPbI}_{3}$ thin films. (a) (Top) Optical image of a measurement chip including a thin film. (Bottom) SEM image of the measurement area (scalebar is $200 \mu \mathrm{m}$ ). The red dashed squares are the measurement areas (membranes). In the middle of membrane is the heating stripe. The larger membrane is used for the primary measurement, whilst the smaller membrane is used for the heat loss correction due to radiation. (b) Temperature dependence of thermal conductivity of vapour deposited thin films from $100 \mathrm{~K}$ to $300 \mathrm{~K} .65 \mathrm{~nm}, 80 \mathrm{~nm}$ and $100 \mathrm{~nm}$ thick films corresponded to black, red and blue dots, respectively. (c) SEM images of perovskite films deposited by AACVD method. (d) Temperature-dependent thermal conductivity values from $100 \mathrm{~K}$ to $300 \mathrm{~K}$ of perovskite films deposited by AACVD method. The red and blue lines are fits to the data for orthorhombic and tetragonal phases respectively to enable visualisation of the discontinuity, $\Delta \kappa$, at the phase transition. 

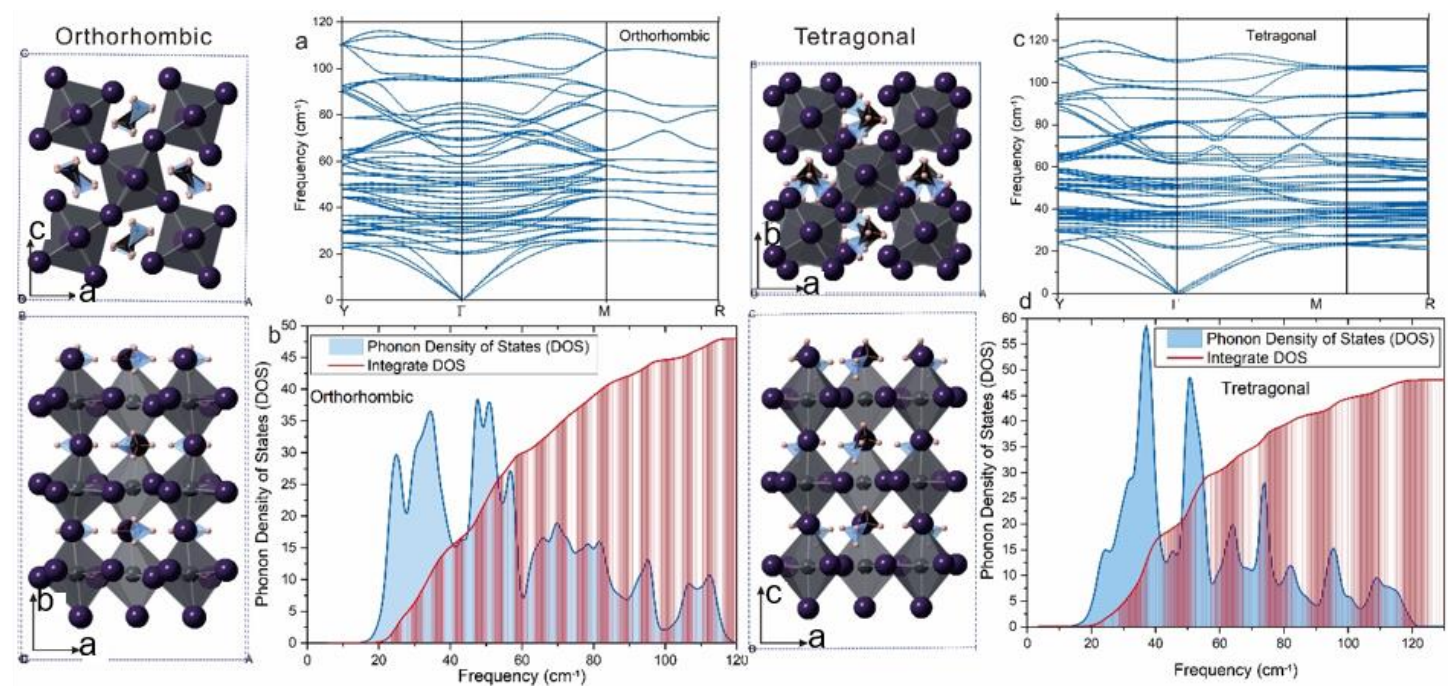

Figure 3. Phonon dispersions (top) and phonon density of states (bottom) of orthorhombic $(a, b)$ and tetragonal $(c, d)$ phases of $\mathrm{MAPbI}_{3}$. The insets show the crystal structure in each phase. 

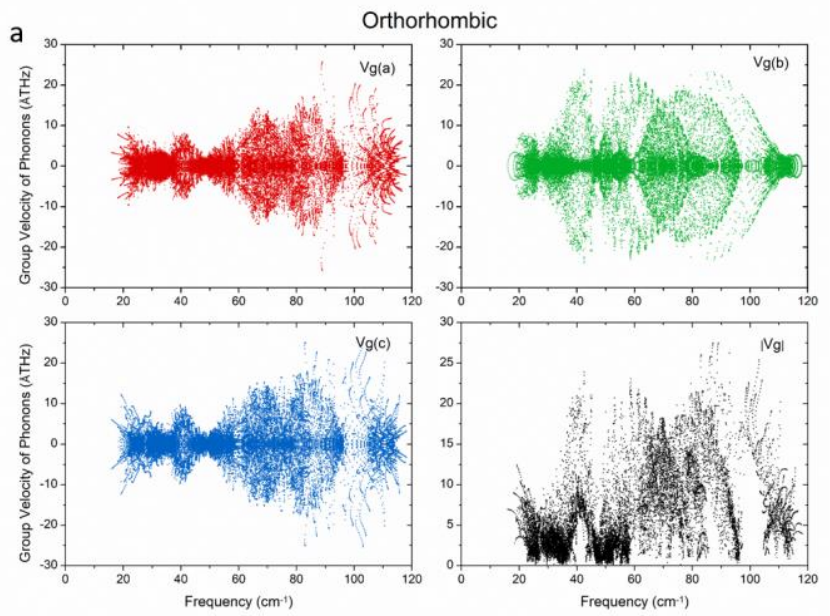

b

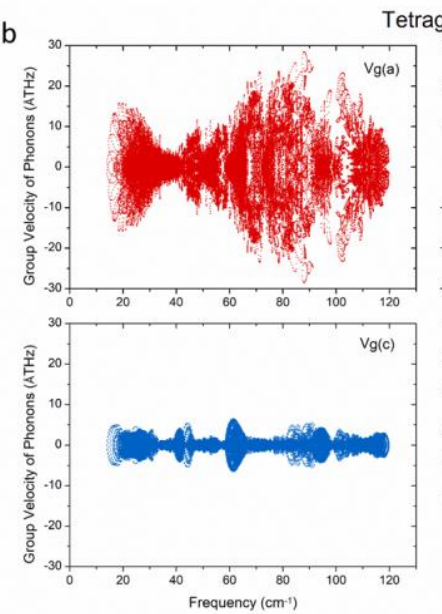

Figure 4. Group velocity of phonons plotted against wavenumber for each crystal axis of the orthorhombic (left) and tetragonal (right) phases of $\mathrm{MAPbI}_{3}$. 
a

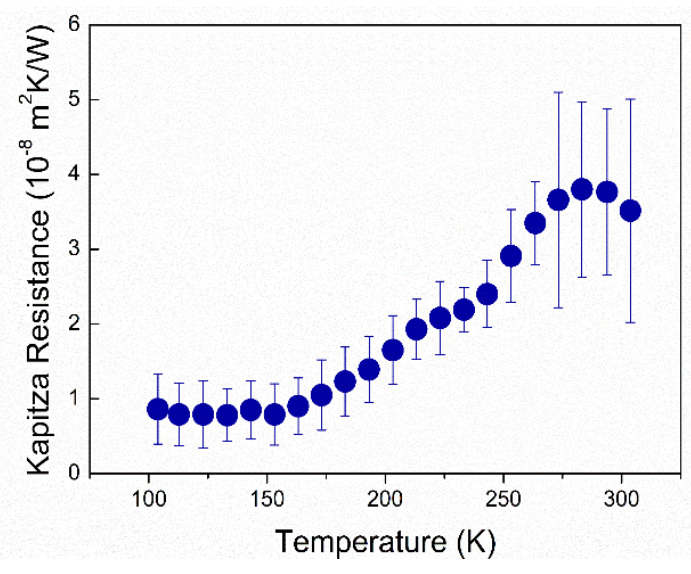

b

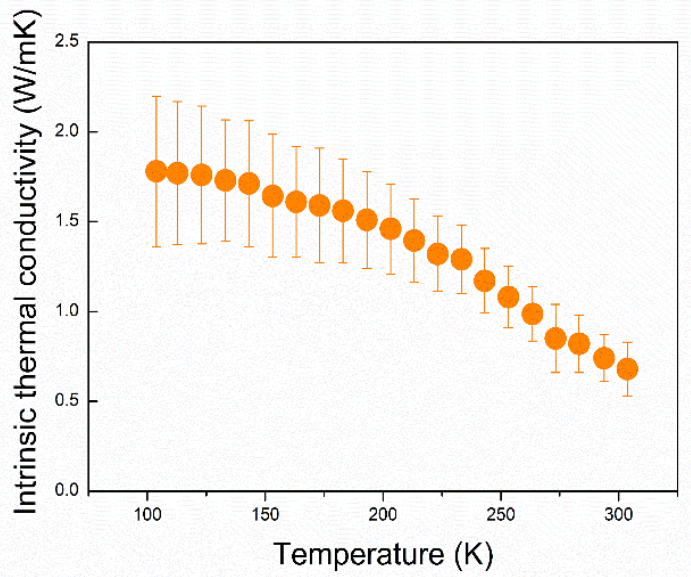

C

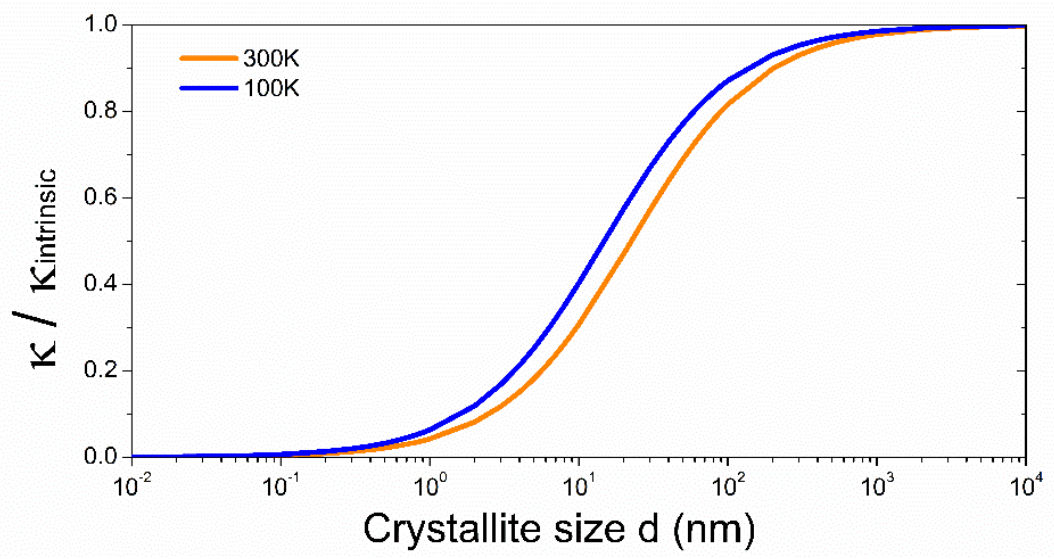

Figure 5. Thermal boundary resistance. (a) Kapitza resistance and (b) intrinsic thermal conductivity derived from the measured thermal conductivity. (c) Predictions for the effect of the crystallite size on the normalized thermal conductivity of perovskites polycrystalline thin films at $100 \mathrm{~K}$ (blue) and $300 \mathrm{~K}$ (orange). 


\section{ASSOCIATED CONTENT}

\section{Supporting Information.}

Supporting Information is availabe from the ACS Publication website at DOI:XXX. The file contains further morphological characterization and crystallography; details of thermal conductivity measurement; and density functional theory (DFT) method.

\section{AUTHOR INFORMATION}

\section{Corresponding Author}

*E-mail: o.fenwick@gmul.ac.uk

\section{Author contributions}

T.L. carried out the experimental study on thermally evaporated films and conducted all thermal measurements, under the supervision of O.F. . S.R. fabricated AACVD perovskite films under the supervision of M. A. M. and J. B.. S.-Y. Y. performed DFT calculations supervised by M.H.. T.D. and P.V. assisted with deposition and morphological characterisation. The manuscript was written through contributions of all authors, and all authors have given approval to the final version of the manuscript.

${ }^{\#}$ T. Liu and S.-Y. Yue made equal contributions to this work.

\section{Notes}

The authors declare no competing financial interest.

\section{ACKNOWLEDGEMENTS}

This work was financed under OF's Royal Society University Research Fellowship, UF140372. T.L. is funded by the China Scholarship Council, and S.R.'s studentship is 
funded by the EPSRC through the Centre for Doctoral Training in Plastic Electronic Materials. M.H. acknowledges the start-up fund from the University of South Carolina. 


\section{REFERENCES}

1. Kojima, A.; Teshima, K.; Shirai, Y.; Miyasaka, T., Organometal Halide Perovskites as Visible-Light Sensitizers for Photovoltaic Cells. J Am Chem Soc 2009, 131 (17), 6050-+.

2. Burschka, J.; Pellet, N.; Moon, S. J.; Humphry-Baker, R.; Gao, P.; Nazeeruddin, M. K.; Gratzel, M., Sequential deposition as a route to high-performance perovskitesensitized solar cells. Nature 2013, 499 (7458), 316-+.

3. Lee, M. M.; Teuscher, J.; Miyasaka, T.; Murakami, T. N.; Snaith, H. J., Efficient Hybrid Solar Cells Based on Meso-Superstructured Organometal Halide Perovskites. Science 2012, 338 (6107), 643-647.

4. Dong, Q. F.; Fang, Y. J.; Shao, Y. C.; Mulligan, P.; Qiu, J.; Cao, L.; Huang, J. S., Electron-hole diffusion lengths $>175 \mathrm{mu} \mathrm{m}$ in solution-grown $\mathrm{CH} 3 \mathrm{NH} 3 \mathrm{PbI} 3$ single crystals. Science 2015, 347 (6225), 967-970.

5. Shi, D.; Adinolfi, V.; Comin, R.; Yuan, M. J.; Alarousu, E.; Buin, A.; Chen, Y.; Hoogland, S.; Rothenberger, A.; Katsiev, K.; Losovyj, Y.; Zhang, X.; Dowben, P. A.; Mohammed, O. F.; Sargent, E. H.; Bakr, O. M., Low trap-state density and long carrier diffusion in organolead trihalide perovskite single crystals. Science 2015, 347 (6221), 519-522.

6. Chen, Y. N.; He, M. H.; Peng, J. J.; Sun, Y.; Liang, Z. Q., Structure and Growth Control of Organic-Inorganic Halide Perovskites for Optoelectronics: From Polycrystalline Films to Single Crystals. Adv. Sci. 2016, 3 (4), 1500392.

7. Liu, M. Z.; Johnston, M. B.; Snaith, H. J., Efficient planar heterojunction perovskite solar cells by vapour deposition. Nature 2013, 501 (7467), 395-398.

8. Senanayak, S. P.; Yang, B. Y.; Thomas, T. H.; Giesbrecht, N.; Huang, W. C.; Gann, E.; Nair, B.; Goedel, K.; Guha, S.; Moya, X.; McNeill, C. R.; Docampo, P.; Sadhanala, A.; Friend, R. H.; Sirringhaus, H., Understanding charge transport in lead iodide perovskite thin-film field-effect transistors. Sci. Adv. 2017, 3 (1), e1601935.

9. Matsushima, T.; Yasuda, T.; Fujita, K.; Adachi, C., Field-effect transistors with vacuum-deposited organic-inorganic perovskite films as semiconductor channels. $J$. Appl. Phys. 2016, 120 (23), 233301.

10. Zhang, L. Q.; Yang, X. L.; Jiang, Q.; Wang, P. Y.; Yin, Z. G.; Zhang, X. W.; Tan, H. R.; Yang, Y.; Wei, M. Y.; Sutherland, B. R.; Sargent, E. H.; You, J. B., Ultra-bright and highly efficient inorganic based perovskite light-emitting diodes. Nat. Commun. 2017, 8, 15640.

11. Tan, Z. K.; Moghaddam, R. S.; Lai, M. L.; Docampo, P.; Higler, R.; Deschler, F.; Price, M.; Sadhanala, A.; Pazos, L. M.; Credgington, D.; Hanusch, F.; Bein, T.; Snaith, H. J.; Friend, R. H., Bright light-emitting diodes based on organometal halide perovskite. Nat. Nanotechnol. 2014, 9 (9), 687-692.

12. Ling, Y. C.; Yuan, Z.; Tian, Y.; Wang, X.; Wang, J. C.; Xin, Y.; Hanson, K.; Ma, B. W.; Gao, H. W., Bright Light-Emitting Diodes Based on Organometal Halide Perovskite Nanoplatelets. Adv. Mater. 2016, 28 (2), 305-311. 
13. Lee, W.; Li, H. S.; Wong, A. B.; Zhang, D. D.; Lai, M. L.; Yu, Y.; Kong, Q.; Lin, E.; Urban, J. J.; Grossman, J. C.; Yang, P. D., Ultralow thermal conductivity in allinorganic halide perovskites. P Natl Acad Sci USA 2017, 114 (33), 8693-8697.

14. Mettan, X.; Pisoni, R.; Matus, P.; Pisoni, A.; Jaamovic, J.; Nafradi, B.; Spina, M.; Pavuna, D.; Forro, L.; Horvath, E., Tuning of the Thermoelectric Figure of Merit of CH3NH3MI3 (M=Pb,Sn) Photovoltaic Perovskites. J Phys Chem C 2015, 119 (21), 11506-11510.

15. Ye, T.; Wang, X. Z.; Li, X. Q.; Yan, A. Q.; Ramakrishna, S.; Xu, J. W., Ultra-high Seebeck coefficient and low thermal conductivity of a centimeter-sized perovskite single crystal acquired by a modified fast growth method. J. Mater. Chem. C 2017, 5 (5), 1255-1260.

16. Herz, L. M., Charge-Carrier Mobilities in Metal Halide Perovskites: Fundamental Mechanisms and Limits. ACS Energy Lett. 2017, 2 (7), 1539-1548.

17. Elbaz, G. A.; Ong, W. L.; Doud, E. A.; Kim, P.; Paley, D. W.; Roy, X.; Malen, J. A., Phonon Speed, Not Scattering, Differentiates Thermal Transport in Lead Halide Perovskites. Nano Lett. 2017, 17 (9), 5734-5739.

18. Lu, N. D.; Li, L.; Liu, M., A review of carrier thermoelectric-transport theory in organic semiconductors. Phys. Chem. Chem. Phys. 2016, 18 (29), 19503-19525.

19. Hata, T.; Giorgi, G.; Yamashita, K., The Effects of the Organic-Inorganic Interactions on the Thermal Transport Properties of CH3NH3PbI3. Nano Lett 2016, 16 (4), 2749-2753.

20. Wang, M. C.; Lin, S. C., Anisotropic and Ultralow Phonon Thermal Transport in Organic-Inorganic Hybrid Perovskites: Atomistic Insights into Solar Cell Thermal Management and Thermoelectric Energy Conversion Efficiency. Adv. Funct. Mater. 2016, 26 (29), 5297-5306.

21. Yue, S. Y.; Zhang, X. L.; Qin, G. Z.; Yang, J. Y.; Hu, M., Insight into the collective vibrational modes driving ultralow thermal conductivity of perovskite solar cells. Phys. Rev. B 2016, 94 (11), 115427.

22. Lee, W.; Li, H.; Wong, A. B.; Zhang, D.; Lai, M.; Yu, Y.; Kong, Q.; Lin, E.; Urban, J. J.; Grossman, J. C.; Yang, P., Ultralow thermal conductivity in all-inorganic halide perovskites. Proceedings of the National Academy of Sciences 2017, 114 (33), 86938697.

23. Abdelhady, A. L.; Saidaminov, M. I.; Murali, B.; Adinolfi, V.; Voznyy, O.; Katsiev, K.; Alarousu, E.; Comin, R.; Dursun, I.; Sinatra, L.; Sargent, E. H.; Mohammed, O. F.; Bakr, O. M., Heterovalent Dopant Incorporation for Bandgap and Type Engineering of Perovskite Crystals. J. Phys. Chem. Lett. 2016, 7 (2), 295-301.

24. Haque, M. A.; Nugraha, M. I.; Paleti, S. H. K.; Baran, D., Role of Compositional Tuning on Thermoelectric Parameters of Hybrid Halide Perovskites. J. Phys. Chem. C 2019, 123 (24), 14928-14933.

25. Zhu, Y. J.; Wang, H. Y.; Wang, B. H.; Liu, X. L.; Wu, H. J.; Licht, S., Solar thermoelectric field plus photocatalysis for efficient organic synthesis exemplified by toluene to benzoic acid. Appl. Catal. B - Environ. 2016, 193, 151-159.

26. Wang, L.; Liu, F. J.; Liu, T. J.; Wang, J. W.; Cai, X. Y.; Wang, G. T.; Ma, T. L.; Jiang, C., Pinhole-Free Perovskite Films by Methylamine Iodide Solution-Assisted 
Repair for High-Efficiency Photovoltaics under Ambient Conditions. ACS Appl. Mater. Inter. 2016, 8 (45), 30920-30925.

27. Maculan, G.; Sheikh, A. D.; Abdelhady, A. L.; Saidaminov, M. I.; Hague, M. A.; Murali, B.; Alarousu, E.; Mohammed, O. F.; Wu, T.; Bakr, O. M., CH3NH3PbCl3 Single Crystals: Inverse Temperature Crystallization and Visible-Blind UVPhotodetector. J. Phys. Chem. Lett. 2015, 6 (19), 3781-3786.

28. Pisoni, A.; Jacimovic, J.; Barisic, O. S.; Spina, M.; Gaal, R.; Forro, L.; Horvath, E., Ultra-Low Thermal Conductivity in Organic-Inorganic Hybrid Perovskite CH3NH3PbI3. J. Phys. Chem. Lett. 2014, 5 (14), 2488-2492.

29. Guo, Z.; Yoon, S. J.; Manser, J. S.; Kamat, P. V.; Luo, T. F., Structural Phase- and Degradation-Dependent Thermal Conductivity of CH3NH3PbI3 Perovskite Thin Films. J. Phys. Chem. C 2016, 120 (12), 6394-6401.

30. Silva, T. S.; Alves, A. S.; Pepe, I.; Tsuzuki, H.; Nakamura, O.; Neto, M. M. F. D.; da Silva, A. F.; Veissid, N.; An, C. Y., Thermal diffusivity of lead iodide. J. Appl. Phys. 1998, 83 (11), 6193-6195.

31. Chen, S.; Briscoe, J.; Shi, Y.; Chen, K.; Wilson, R. M.; Dunn, S.; Binions, R., A simple, low-cost CVD route to high-quality $\mathrm{CH} 3 \mathrm{NH} 3 \mathrm{PbI} 3$ perovskite thin films. CrystEngComm 2015, 17 (39), 7486-7489.

32. Togo, A.; Tanaka, I., First principles phonon calculations in materials science. Scripta Mater. 2015, 108, 1-5.

33. Perdew, J. P.; Burke, K.; Ernzerhof, M., Generalized gradient approximation made simple. Phys. Rev. Lett. 1996, 77 (18), 3865-3868.

34. Blochl, P. E., Projector Augmented-Wave Method. Phys. Rev. B 1994, 50 (24), 17953-17979.

35. Kresse, G.; Joubert, D., From ultrasoft pseudopotentials to the projector augmented-wave method. Phys. Rev. B 1999, 59 (3), 1758-1775.

36. Tkatchenko, A.; Scheffler, M., Accurate Molecular Van Der Waals Interactions from Ground-State Electron Density and Free-Atom Reference Data. Phys. Rev. Lett. 2009, 102 (7), 073005.

37. Yue, S. Y.; Zhang, X. L.; Qin, G. Z.; Yang, J. Y.; Hu, M., Insight into the collective vibrational modes driving ultralow thermal conductivity of perovskite solar cells. Phys. Rev. B 2016, 94 (11).

38. Leguy, A. M. A.; Goni, A. R.; Frost, J. M.; Skelton, J.; Brivio, F.; RodriguezMartinez, X.; Weber, O. J.; Pallipurath, A.; Alonso, M. I.; Campoy-Quiles, M.; Weller, M. T.; Nelson, J.; Walsh, A.; Barnes, P. R. F., Dynamic disorder, phonon lifetimes, and the assignment of modes to the vibrational spectra of methylammonium lead halide perovskites. Phys. Chem. Chem. Phys. 2016, 18 (39), 27051-27066.

39. Maiti, A.; Mahan, G. D.; Pantelides, S. T., Dynamical simulations of nonequilibrium processes - Heat flow and the Kapitza resistance across grain boundaries. Solid State Commun. 1997, 102 (7), 517-521.

40. Hasselman, D. P. H.; Donaldson, K. Y.; Thomas, J. R.; Brennan, J. J., Thermal conductivity of vapor liquid solid and vapor solid silicon carbide whisker-reinforced lithium aluminosilicate glass ceramic composites. J. Am. Ceram. Soc. 1996, 79 (3), 742-748. 
41. Limarga, A. M.; Clarke, D. R., The grain size and temperature dependence of the thermal conductivity of polycrystalline, tetragonal yttria-stabilized zirconia. Appl. Phys. Lett. 2011, 98 (21).

42. Braginsky, L.; Shklover, V.; Hofmann, H.; Bowen, P., High-temperature thermal conductivity of porous Al2O3 nanostructures. Phys. Rev. B 2004, 70 (13), 134201.

43. Nan, C. W.; Birringer, R., Determining the Kapitza resistance and the thermal conductivity of polycrystals: A simple model. Phys. Rev. B 1998, 57 (14), 8264-8268.

44. Nan, C. W.; Birringer, R.; Clarke, D. R.; Gleiter, H., Effective thermal conductivity of particulate composites with interfacial thermal resistance. J. Appl. Phys. 1997, 81 (10), 6692-6699.

45. Bhardwaj, A.; Chauhan, N. S.; Misra, D. K., Significantly enhanced thermoelectric figure of merit of p-type $\mathrm{Mg} 3 \mathrm{Sb}$ 2-based Zintl phase compounds via nanostructuring and employing high energy mechanical milling coupled with spark plasma sintering. $J$. Mater. Chem. A 2015, 3 (20), 10777-10786.

46. Toprak, M. S.; Stiewe, C.; Platzek, D.; Williams, S.; Bertini, L.; Muller, E. C.; Gatti, C.; Zhang, Y.; Rowe, M.; Muhammed, M., The impact of nanostructuring on the thermal conductivity of thermoelectric CoSb3. Adv. Funct. Mater. 2004, 14 (12), 11891196.

47. Stoner, R. J.; Maris, H. J., Kapitza Conductance and Heat-Flow between Solids at Temperatures from 50 to 300 K. Phys. Rev. B 1993, 48 (22), 16373-16387.

48. Smith, D. S.; Fayette, S.; Grandjean, S.; Martin, C.; Telle, R.; Tonnessen, T., Thermal resistance of grain boundaries in alumina ceramics and refractories. J. Am. Ceram. Soc. 2003, 86 (1), 105-111.

49. Kovalsky, A.; Wang, L. L.; Marek, G. T.; Burda, C.; Dyck, J. S., Thermal Conductivity of $\mathrm{CH} 3 \mathrm{NH} 3 \mathrm{PbI} 3$ and $\mathrm{CsPbl}(3)$ : Measuring the Effect of the Methylammonium Ion on Phonon Scattering. J Phys Chem C 2017, 121 (6), 3228-3233. 50. Dou, L. T.; Wong, A. B.; Yu, Y.; Lai, M. L.; Kornienko, N.; Eaton, S. W.; Fu, A.; Bischak, C. G.; Ma, J.; Ding, T. N.; Ginsberg, N. S.; Wang, L. W.; Alivisatos, A. P.; Yang, P. D., Atomically thin two-dimensional organic-inorganic hybrid perovskites. Science 2015, 349 (6255), 1518-1521.

51. Schmidt, L. C.; Pertegas, A.; Gonzalez-Carrero, S.; Malinkiewicz, O.; Agouram, S.; Espallargas, G. M.; Bolink, H. J.; Galian, R. E.; Perez-Prieto, J., Nontemplate Synthesis of CH3NH3PbBr3 Perovskite Nanoparticles. J. Am. Chem. Soc. 2014, 136 (3), 850-853.

52. He, Y.; Galli, G., Perovskites for Solar Thermoelectric Applications: A First Principle Study of CH3NH3AI3 (A = Pb and Sn). Chemistry of Materials 2014, 26 (18), 5394-5400.

53. Volklein, F., Thermal-Conductivity and Diffusivity of a Thin-Film Sio2-Si3n4 Sandwich System. Thin Solid Films 1990, 188 (1), 27-33.

54. Volklein, F.; Reith, H.; Meier, A., Measuring methods for the investigation of inplane and cross-plane thermal conductivity of thin films. Phys. Status. Solidi. A 2013, 210 (1), 106-118.

55. Linseis, V.; Volklein, F.; Reith, H.; Nielsch, K.; Woias, P., Advanced platform for the in-plane ZT measurement of thin films. Rev. Sci. Instrum. 2018, 89 (1). 
Table of contents entry

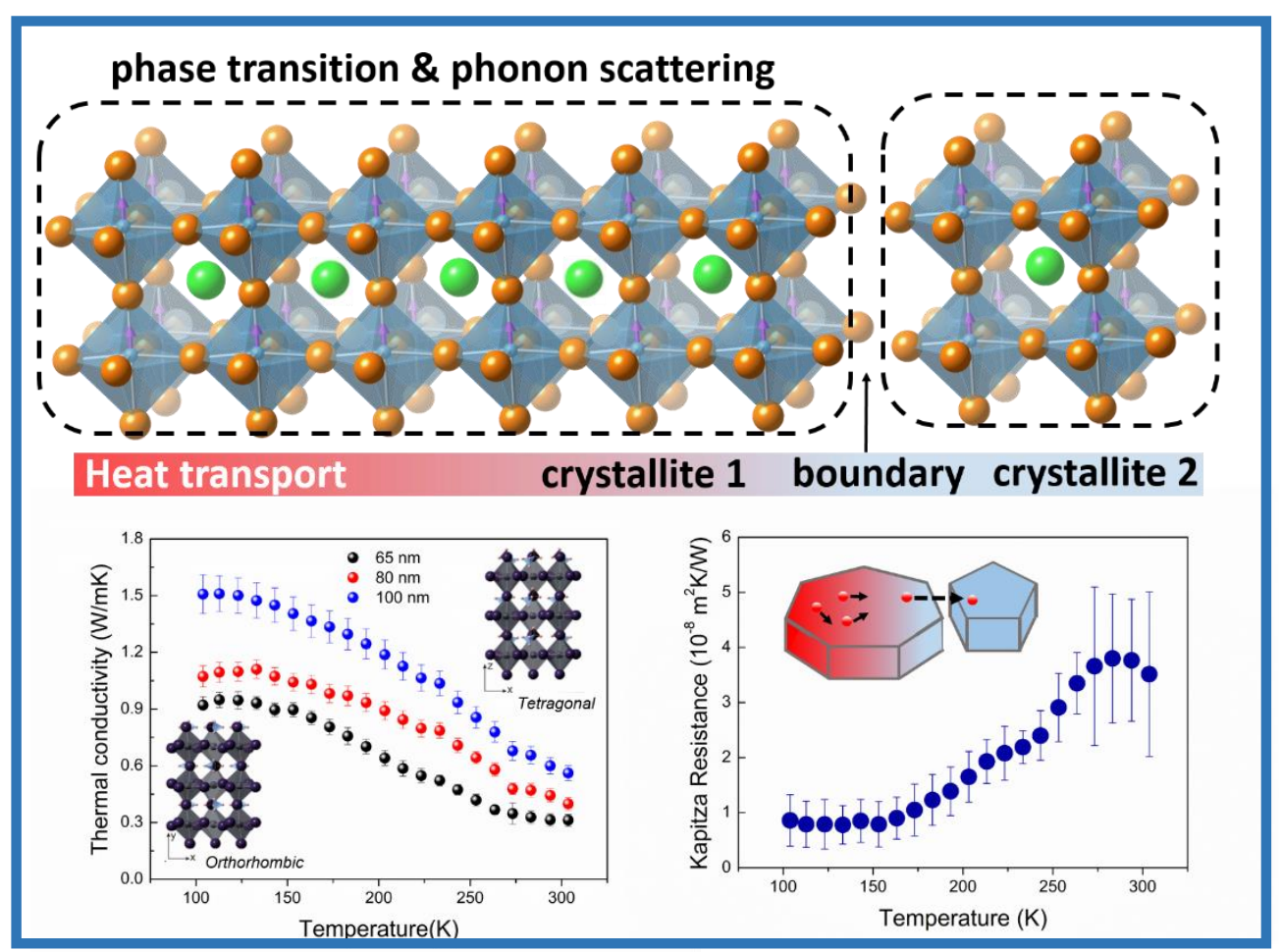

A case study of in-plane thermal transport in methylamonium lead iodide $\left(\mathrm{MAPbI}_{3}\right)$ perovskite thin films.

KEYWORDS: Halide perovskite, phonon, in-plane, thermal conductivity, thermal transport, thermoelectrics. 